\title{
One-dimensional models in turbine blades dynamics
}

\author{
Vladimir V. Eliseev', Artem A. Moskalets², Evgenii A. Oborin ${ }^{3}$
}

\begin{abstract}
Turbine blades are considered as straight naturally twisted rods. Two models are discussed: Bernoulli-Euler beam and Cosserat rod. Linear theories with the small displacements, rotations and loads are used. The equations of dynamics taking into account bending, twisting, axial and shear deformations and cross links between them are derived. The stiffness coefficients in elasticity relations are defined. In the case of harmonic oscillations, we have for amplitudes the ordinary differential equations solved by means of computer mathematics (Mathcad). As a result normal modes, natural frequencies and also the amplitudes of forced oscillations are obtained. For the Bernoulli-Euler beam the LagrangeRitz-Kantorovich variational approach with the approximations of deflections is proposed. The unknown coefficients of approximation depending on time are found by the numerical integration of the Lagrange system of equations. Proposed methods are applied to the calculation of the real turbine blade.
\end{abstract}

Keywords Turbine blades, Naturally twisted rods, Linear rod theory, Elastic modulus, Natural frequencies, Normal modes, Variational approach, Forced oscillations, Computer mathematics.

\section{Introduction}

The analysis of turbine blades stress-strain state continues to attract attention of mechanical engineers [1-7], but the focuses in that area are shifted. Initially turbine blades calculations as the beams [8-10] predominated, but then the finite element analysis of the three-dimensional models began to displace them [11]. However, the scopes of one-dimensional models are not exhausted. With them the

\author{
${ }^{1}$ V.V. Eliseev \\ Peter the Great Saint-Petersburg Polytechnic University, SPb, Russia \\ e-mail: yeliseyev@inbox.ru \\ ${ }^{2}$ A.A. Moskalets \\ e-mail: artem.moskalec@gmail.com \\ ${ }^{3}$ E.A. Oborin \\ e-mail: oborin1@yandex.ru
}


problems unavailable in the three-dimensional formulation [12, 13] can be solved. Considering the rod models are justified if the peculiar properties of the threedimensional stress-strain state are not appeared [14, 15].

The goal of this work is the creation of the engineering methods of turbine blade calculation with the two one-dimensional models [16, 17]: Bernoulli-Euler beam (with the bending in two planes) and Cosserat rod. For the beam the variational method of Lagrange [18] with the combination of computer mathematics [19] is used, it allows us to solve also some difficult nonlinear oscillation problems [17].

The full one-dimensional model of the turbine blade as the twisted rod [20-22] taking into account bending, twisting and axial deformations as interconnected is more complex. The cross link between bending and twisting arises from the difference between the center of stiffness and the center of mass. The cross link between extension and twisting arises from the natural twisting. The analysis of such uneasy model became possible due to the progress of elastic rod mechanics $[18,23-25]$ and computer mathematics.

\section{Blade as the beam with bending in two planes}

We involve the triple of Cartesian axes directing the $\mathrm{z}$-axis through the cross section centers of mass (we assume that the centers of mass lie on the same line). The displacement of twisted rod always has two components $u_{x}, u_{y}$, that are the functions of coordinate $z$ and time $t$. The kinetic energy is the integral along the blade length:

$$
T=1 / 2 \int_{0}^{l} \rho\left(\dot{u}_{x}^{2}+\dot{u}_{y}^{2}\right) d z
$$

where $\rho$ is the mass distributed per unit length.

We define the potential energy with the usual for the beam theory assumption, that the axial displacement at the bending is $u_{z}=-x u_{x}^{\prime}-y u_{y}^{\prime}$ (here prime is the derivative with respect to $z$ ):

$$
\Pi=E / 2 \int_{0}^{l} d z \int_{F} u_{z}^{\prime 2} d F=E / 2 \int_{0}^{l}\left(J_{x} u_{x}^{\prime \prime 2}+2 J_{x y} u_{x}^{\prime \prime} u_{y}^{\prime \prime}+J_{y} u_{y}^{\prime \prime 2}\right) d z
$$

(here $E$ is the Young's modulus). We involve the moments of inertia as integrals $J_{x}=\int x^{2} d F, J_{x y}=\int x y d F, J_{y}=\int y^{2} d F$ over the cross section $F$.

From the model with distributed parameters we proceed to the discrete one by means of displacements approximation

$$
u_{x}(z, t)=\sum_{k=1}^{n} U_{x k}(t) \varphi_{k}(z)=U_{x}(t)^{T} \varphi(z), u_{y}(z, t)=U_{y}(t)^{T} \varphi(z)
$$

(with the matrix notation). The functions $U_{x, y}(t)$ characteristic to the Kantorovich method [18] play the role of generalized coordinates and are the 
subjects to the definition by the Lagrange equation system. The coordinate functions $\varphi(z)$ are given according to the boundary conditions (fixed at $z=0$ ). We accept

$$
\varphi_{i}(z)=z^{1+i}, i=1, \ldots, n
$$

Substituting the formulas (1.3) and (1.4) into the definitions (1.1) and (1.2), we obtain the kinetic energy and the potential energy of the blade discrete model

$$
\begin{aligned}
& T=1 / 2\left(\dot{U}_{x}^{T} m \dot{U}_{x}+\dot{U}_{y}^{T} m \dot{U}_{y}\right), m=\rho \int_{0}^{l} \varphi \varphi^{T} d x ; \\
& \Pi=1 / 2\left(U_{x}^{T} C_{x} U_{x}+2 U_{x}^{T} C_{x y} U_{y}+U_{y}^{T} C_{y} U_{y}\right), \\
& C_{x}=E \int_{0}^{l} J_{x} \varphi^{\prime \prime} \varphi^{\prime \prime T} d z, C_{x y}=E \int_{0}^{l} J_{x y} \varphi^{\prime \prime} \varphi^{\prime \prime T} d z, C_{y}=E \int_{0}^{l} J_{y} \varphi^{\prime \prime} \varphi^{\prime \prime T} d z
\end{aligned}
$$

with the stiffness matrix and the inertia matrix

The generalized forces for the Lagrange equations are found by the virtual work:

$$
\int_{0}^{l}\left(f_{x} \delta u_{x}+f_{y} \delta u_{y}\right) d z=Q_{x}^{T} \delta U_{x}+Q_{y}^{T} \delta U_{y}, Q_{x}=\int_{0}^{l} f_{x} \varphi d z .
$$

The column $Q_{y}$ is founded similarly. Here $f_{x}, f_{y}$ are the components of the distributed per unit length load.

\section{The Lagrange equations and solving of them}

Reducing the notation (1.1-1.3) by involving block columns and block matrixes

$$
U=\left(\begin{array}{l}
U_{y} \\
U_{z}
\end{array}\right), M=\left(\begin{array}{cc}
m & 0 \\
0 & m
\end{array}\right), C=\left(\begin{array}{cc}
C_{x} & C_{x y} \\
C_{x y} & C_{y}
\end{array}\right), Q=\left(\begin{array}{l}
Q_{x} \\
Q_{y}
\end{array}\right),
$$

we write the Lagrange equations

$$
\left(\frac{\partial K}{\partial \dot{U}}\right)^{\bullet}-\frac{\partial K}{\partial U}=-\frac{\partial \Pi}{\partial U}+Q \Rightarrow M \ddot{U}+C U=Q(t) .
$$

In the case of free oscillations ( $Q=0)$, the main oscillations are considered with the normal modes $\Phi$ and the natural frequencies $\lambda$ :

$$
U(t)=\Phi \sin \lambda t ;\left(C-\lambda^{2} M\right) \Phi=0 .
$$

Here is the generalized eigenvalue problem solved by the Mathcad built-in functions [19].

The normal modes $\Phi_{i}$ have the well-known properties of orthogonality. Normalizing the modes we obtain $\Phi_{i}^{T} M \Phi_{k}=\delta_{i k}, \Phi_{i}^{T} C \Phi_{k}=\lambda_{i}^{2} \delta_{i k}$. Expanding the displacement according to the modes, we arrive to the main coordinates $V_{i}$ : 


$$
U(t)=\sum_{i=1}^{2 n} V_{i}(t) \Phi_{i}=\Gamma V, \Gamma=\left(\Phi_{1} \ldots \Phi_{n}\right)
$$

The basis matrix $\Gamma$ is composed of the mode columns. With the main coordinates we write

$$
K=\frac{1}{2} \sum \dot{V}_{i}^{2}, \Pi=\frac{1}{2} \sum \lambda_{i}^{2} V_{i}^{2}, \underline{V_{i}+\lambda_{i}^{2} V_{i}=P_{i}(t)}, P=\Gamma^{T} Q
$$

The underlined equation is solved with the Duhamel's integral

$$
V_{i}=V_{i}(0) \cos \lambda_{i} t+\dot{V}_{i}(0) \lambda_{i}^{-1} \sin \lambda_{i} t+\lambda_{i}^{-1} \int_{0}^{t} P_{i}(\tau) \sin \lambda_{i}(t-\tau) d \tau
$$

The formulas (1.12), (1.10) and (1.3) give the solution of the forced oscillation problem if the frequencies and modes are known.

The calculation is simplified if computer mathematics (Mathcad) is utilized. The set of frequencies and the basis matrix $\Gamma$ are found by the built-in functions genvals and genvecs.

The calculations of the specific blade produced by one the companies of Russia are done. The length of the blade $l=0,78 \mathrm{~m}$, the cross section is presented in fig. 1. The cross section profile is bounded by the graphs of two functions $y_{l}(x), y_{t}(x)$ defined by points with regression (the built-in functions regress and interp):

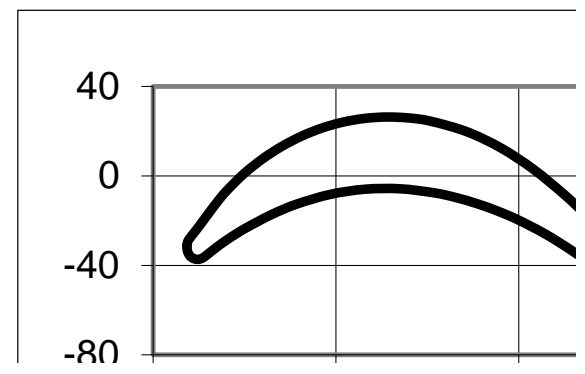

Fig. 1. The blade cross section in $\mathrm{mm}$

The approximation of displacements (1.3) with the number of terms $n=5$ is accepted. Then the number of the degrees of freedom, of the frequencies and of the modes is $2 n=10$. In fig. 2 the results for the first six modes are presented:

Further we discuss the example of calculation of transient forced oscillations. The distributed per unit length loads are given by

$$
f_{x, y}(z, t)=f_{x, y}(z) \cos (\omega(t) t), \omega(t)=\lambda_{0}+\dot{\lambda} t
$$

where $f_{x, y}(z)$ are the presented blade aerodynamics results, and $\lambda_{0}, \dot{\lambda}$ are given constants. We have the abruptly applied load with the pass through the resonance, and $\lambda_{0}$ is less than one of natural frequencies. The calculation according the above discussed method does not cause difficulties, and the integrals (1.12) are computed by means of Mathcad. 

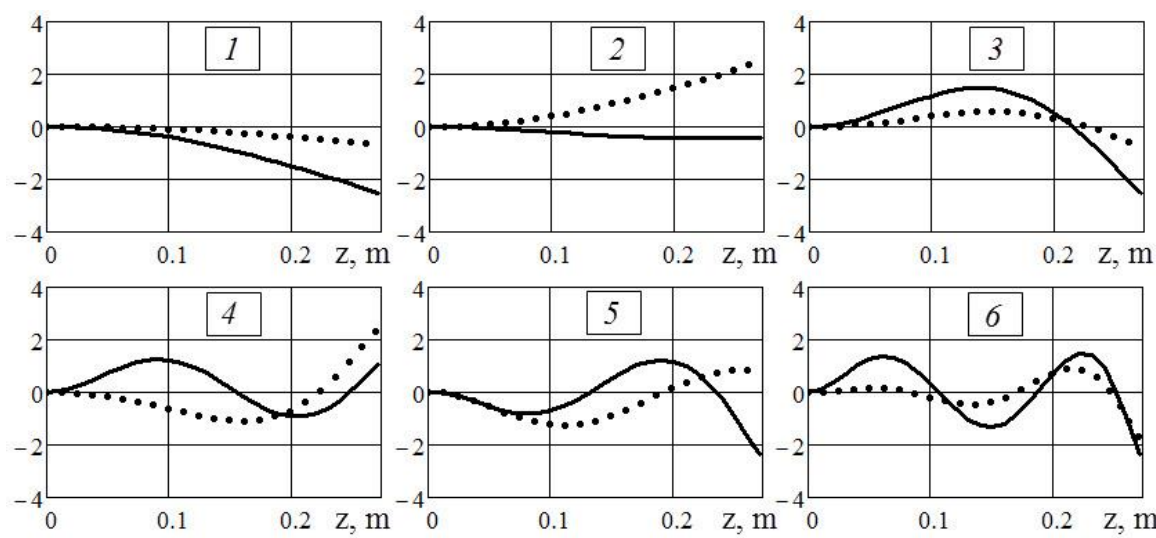

$\mathrm{u}_{\mathrm{xn}}(\mathrm{z})$

$u_{y n}(z)$

Fig. 2. The normal modes

Note, that due to the computer mathematics the preliminary calculation of the frequencies and the modes ceases to be compulsory - the system of ordinary differential equations (1.8) (ODE) can be integrated right off. We represent this system

$$
\dot{U}=v, \dot{v}=M^{-1}(Q-C U)
$$

and appeal to the built-in function Radau. This function is the special numerical method for solving the stiff systems of ODE [19]. The conventional Runge-Kutta methods turn out to be ineffective for the system (1.14). In fig. 3 results for the pass through the first resonance with $f_{x}(z)=1000=-f_{y}(z), \lambda_{0}=470, \dot{\lambda}=2$ are presented (in $\mathrm{m}$ ). The amplitude of the oscillations $\approx 4 \mathrm{~cm}-$ such large deformations are not allowed.

Note that considering the forced harmonic oscillations, the system (1.9) for the amplitudes becomes nonhomogeneous and is solved by the built-in function lsolve.

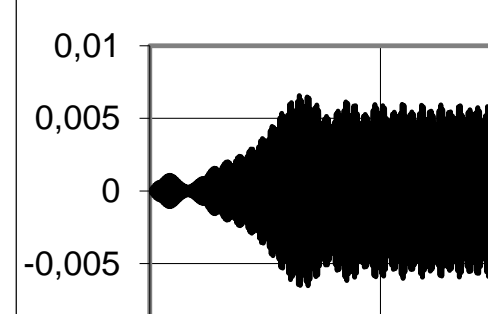

$u_{x}(l, t)$

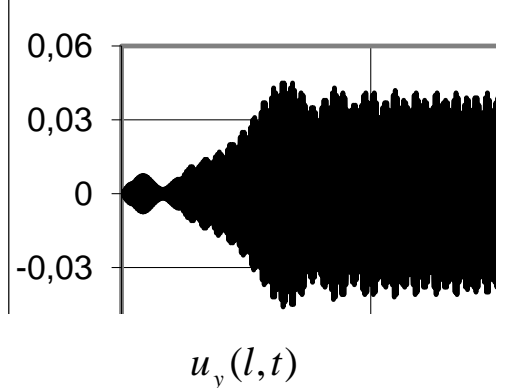

Fig. 3. The transient oscillations 


\section{The full one-dimensional rod model}

The Cosserat rods are represented as material lines for which particles the displacement vector $\mathbf{u}(z, t)$ and the small rotation vector $\boldsymbol{\theta}(z, t)$ (as the functions of coordinate and time) are given. Interactions are expressed by the force vector $\mathbf{Q}(z, t)$ and the moment vector $\mathbf{M}(z, t)$. The full system of equations of the linear elastic rod mechanics has the form [18]:

$$
\begin{aligned}
& \mathbf{Q}^{\prime}+\mathbf{q}=\rho \ddot{\mathbf{u}}, \mathbf{M}^{\prime}+\mathbf{k} \times \mathbf{Q}+\mathbf{m}=\mathbf{G} \cdot \ddot{\boldsymbol{\theta}}, \\
& \boldsymbol{\theta}^{\prime}=\mathbf{A} \cdot \mathbf{M}+\mathbf{C} \cdot \mathbf{Q}, \mathbf{u}^{\prime}=\boldsymbol{\theta} \times \mathbf{k}+\mathbf{B} \cdot \mathbf{Q}+\mathbf{M} \cdot \mathbf{C} .
\end{aligned}
$$

The first two equations express the laws of balance of momentum and angular momentum. In them $\rho$ is the distributed per unit length mass, $\mathbf{G}$ is the inertia tensor, $\mathbf{k}$ is the tangent unit vector (z-axes). The third and the fourth equations are the elasticity relations connecting the deformation vectors $\boldsymbol{\theta}^{\prime}$ and $\boldsymbol{\gamma}=\mathbf{u}^{\prime}-\boldsymbol{\theta} \times \mathbf{k}$ with the force factors (the stress resultants). Note that the deformation vector $\boldsymbol{\theta}^{\prime}$ defines curving and twisting of the rod, and the vector $\gamma$ defines extension (compression) and transversal shear. In elasticity relations we have three tensors of compliance: $\mathbf{A}$ is the compliance in bending and twisting, $\mathbf{B}$ is the compliance in tension and shear and $\mathbf{C}$ is the tensor of cross links.

The distributed per unit length force $\mathbf{q}$ and moment $\mathbf{m}$ are the applied external loads in (1.15). They are assumed to be small in the considered linear theory as like as $\mathbf{u}, \boldsymbol{\theta}, \mathbf{Q}, \mathbf{M}$. Then $\mathbf{G}, \mathbf{A}, \mathbf{B}, \mathbf{C}$ correspond the initial (unstrained) state and do not depend on time.

The tensors of compliance $\mathbf{A}, \mathbf{B}, \mathbf{C}$ are founded by means of the theory of elasticity from the solution of Saint-Venant problem or (that is more difficult) from the asymptotic analysis of the three-dimensional problem with small thickness [19]. The calculation of the compliance tensors is a separate topic beyond the scope of this work.

The model with the equations (1.15) is called full because it describes bending, twisting and extension of the rod as interconnected deformations. Defined by tensor $\mathbf{C}$ cross links arise from shifting of the mass center and from natural twisting. The consequence of this links is the simultaneous bending, twisting and tension of the blade practically under any load.

Further the full one-dimensional blade model, a method of calculation using the computer mathematics and the estimation of the links influence on the stress-strain state are presented.

The principal directions of the inertia tensor $\mathbf{G}$ are rotated relative to initial ones (with $z=0$ ) by an angle $\varphi(z)$. The twist $\Omega=\varphi^{\prime}(z)$ is involved. For the straight rod from the generalized Saint-Venant problem, it is established [22]:

$$
\mathbf{A}=(E \mathbf{I})^{-1}+A_{z} \mathbf{k k}, \mathbf{B}=(\mu F \mathbf{K})^{-1}+B_{z} \mathbf{k k}, \mathbf{C}=A_{z} \mathbf{k} \boldsymbol{\eta}+C_{z} \mathbf{k} \mathbf{k}
$$


Here $\mathbf{J}=\int \mathbf{x} \mathbf{x} d F, J=\operatorname{tr} \mathbf{J}, \mathbf{I}=-\mathbf{k} \times \mathbf{J} \times \mathbf{k}$ are the geometrical moments of inertia ( $\mathbf{x}$ is the position vector in the cross section); we see the well-known expressions of compliance in bending $(E \mathbf{I})^{-1}$ and extension (compression) $B_{z}=(E F)^{-1}$.

In relations (1.16) the geometrical twisting stiffness $C_{\Phi}$, the position vector of the center of bending $\boldsymbol{\eta}$ and the coefficient of cross link between extension and twisting $C_{z}$ are included. The definition of them is associated with the solving of problem of twisting $[18,27]$ :

$$
\begin{aligned}
& \Delta \Phi=-2,\left.\Phi\right|_{\partial F}=0 ; \Delta W=0, \partial_{n} W=\mathbf{n} \times \mathbf{x} \cdot \mathbf{k} ; \\
& C_{\Phi}=2 \int \Phi d F, A_{z}=\left(\mu C_{\Phi}\right)^{-1}, \\
& C_{z}=\Omega(\mu F)^{-1}\left(1-J C_{\Phi}^{-1}\right), \\
& \boldsymbol{\eta}=\mathbf{J}^{-1} \cdot \int \mathbf{x} W d F .
\end{aligned}
$$

We can define the warping function $W$ without solving the Neumann boundary value problem (1.17) if the Cauchy-Riemann equations for conjugate harmonic functions are used:

$$
\nabla W=\nabla\left(\Phi+\frac{x^{2}}{2}\right) \times \mathbf{k} \Rightarrow W=\int\left[\left(\partial_{y} \Phi+y\right) d x-\left(\partial_{x} \Phi+x\right) d y\right]
$$

Also in equations (1.16) $\mathbf{K}$ is the tensor of shear factors. Its definition is related to the solving two more boundary value problems for the vector fields in the cross sections [18, 22]. In this article $\mathbf{K}$ is the identity tensor.

To determine the stress function $\Phi$ and the warping function $W$ we used the variational method [27]. As above we introduce two functions $y_{t}(x), y_{l}(x)$ whose graphs bound the cross section top and bottom. We find the approximate solution in the form $\Phi=\alpha\left(y-y_{t}(x)\right)\left(y-y_{l}(x)\right)=\alpha \Phi_{0}$. The unknown varied factor $\alpha$ is founded by the minimization of the functional

$$
J=\int_{F}\left[|\nabla \Phi|^{2}-4 \Phi\right] d F=\int_{x_{0}}^{x_{1}} d x \int_{y_{l}(x)}^{y_{t}(x)}\left(\alpha^{2}\left|\nabla \Phi_{0}\right|^{2}-4 \alpha \Phi_{0}\right) d y \rightarrow \min .
$$

Then the geometric twisting stiffness is $C_{\Phi}=2 \alpha \int_{x_{0}}^{x_{1}} d x \int_{y_{t}(x)}^{y_{t}(x)} \Phi_{0} d y$. Further we define the warping function $W$ according with (1.18):

$$
W(x, y)=A\left(\int_{x_{0}}^{x} \partial_{y} \Phi_{0} d x-\int_{0}^{y} \partial_{x} \Phi_{0} d y\right)-x y
$$

Again the real blade contour is given by the array of points (about 30), and the functions $y_{t}(x), y_{l}(x)$ are defined in Mathcad by the regression (the built-in function regress and interp).

In the projections from the vector equations (1.15) we obtain the system 


$$
\begin{aligned}
& Q_{x}^{\prime}=-q_{x}+\rho \ddot{u}_{x}, Q_{y}^{\prime}=-q_{y}+\rho \ddot{u}_{y}, Q_{z}^{\prime}=-q_{z}+\rho \ddot{u}_{z}, \\
& M_{x}^{\prime}=Q_{y}-m_{x}+G_{x} \ddot{\theta}_{x}+G_{x y} \ddot{\theta}_{y}, \\
& M_{y}^{\prime}=-Q_{x}-m_{y}+G_{y} \ddot{\theta}_{y}+G_{x y} \ddot{\theta}_{x}, \\
& M_{z}^{\prime}=-m_{z}+G_{z} \ddot{\theta}_{z}, \\
& \theta_{x}^{\prime}=A_{x} M_{x}+A_{x y} M_{y}, \theta_{y}^{\prime}=A_{y} M_{y}+A_{x y} M_{x}, \\
& \theta_{z}^{\prime}=A_{z}\left(M_{z}+\eta_{x} Q_{x}+\eta_{y} Q_{y}\right)+C_{z} Q_{z}, \\
& u_{x}^{\prime}=\theta_{y}+B_{x} Q_{x}+B_{x y} Q_{y}+A_{z} \eta_{x} M_{z}, \\
& u_{y}^{\prime}=-\theta_{x}+B_{y} Q_{y}+B_{x y} Q_{x}+A_{z} \eta_{y} M_{z}, \\
& u_{z}^{\prime}=B_{z} Q_{z}+C_{z} M_{z} .
\end{aligned}
$$

Considering the harmonic oscillations with the frequency $\lambda$, variables are changing according to the law $u_{x}(z, t)=u_{x}(z) \sin \lambda t, \ddot{u}_{x}=-\lambda^{2} u_{x}$, and for the amplitudes we obtain the system of ODE of twelfth order.

The coefficients of these equations contain the components of vectors and tensors rotating with the angular velocity $\Omega$ when the coordinate $z$ is increased. The formulas of the components transformation:

$$
\begin{aligned}
& \eta_{x}=\eta_{1} \cos \varphi-\eta_{2} \sin \varphi, \eta_{y}=\eta_{1} \sin \varphi+\eta_{2} \cos \varphi ; \\
& I_{x y}=\frac{I_{1}-I_{2}}{2} \sin 2 \varphi+I_{12} \cos 2 \varphi, \\
& I_{x}=\frac{I_{1}+I_{2}}{2}+\frac{I_{1}-I_{2}}{2} \cos 2 \varphi-I_{12} \sin 2 \varphi, \\
& I_{y}=\frac{I_{1}+I_{2}}{2}-\frac{I_{1}-I_{2}}{2} \cos 2 \varphi+I_{12} \sin 2 \varphi .
\end{aligned}
$$

Substituting (1.21) into (1.20), we obtain the difficult system of ODE with the variable coefficients. However it is not hard to solve by means of computer mathematics (Mathcad). The system (1.20) is represented in the matrix form $Y^{\prime}=D(z, Y)$, where the column $Y$ contains 12 elements: the components of force, moment, rotation angle and displacement (the form of the function $D$ is clear from (1.20)). To the mentioned system of ODE boundary conditions should be added. For the cantilevered turbine blade with the fixed end $z=0$ and another end $z=L$ free from loads, we have the conditions $Y_{9}=Y_{10}=Y_{11}=Y_{6}=Y_{7}=Y_{8}=0 \quad$ and $\quad Y_{0}=Y_{1}=Y_{2}=Y_{3}=Y_{4}=Y_{5}=0$ correspondingly. The formulated boundary value problem is solving by shooting method with the built-in functions sbval and rkfixed.

According to the described above theory, the calculations of the real blade are done with the same parameters as above. The distributions of applied loads are shown in fig. 4:

$$
q_{x}, \mathrm{kN} / \mathrm{m} \quad q_{y}, \mathrm{kN} / \mathrm{m}
$$




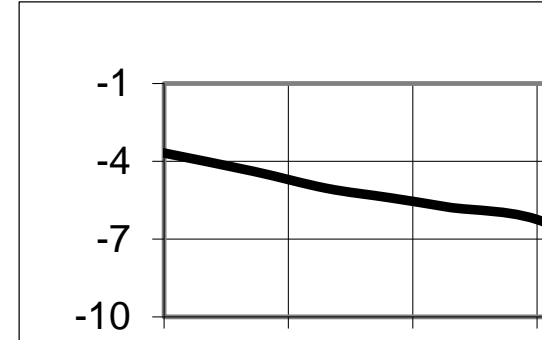

$z, \mathrm{~m}$

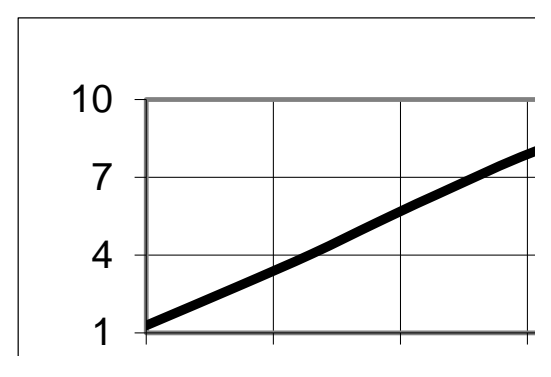

$z, \mathrm{~m}$

Fig. 4. The applied loads

The calculated deflection components $u_{x}, u_{y}$, the angle of rotation $\theta_{z}$ and the axial displacements $u_{z}$ are in fig. 5. Taking into account the later can be important [13], not only because of the centrifugal force.

$u_{x}, \mathrm{~mm}$

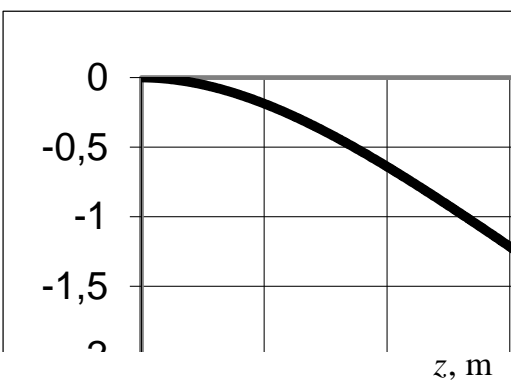

$\theta_{z}, 10^{-4}$

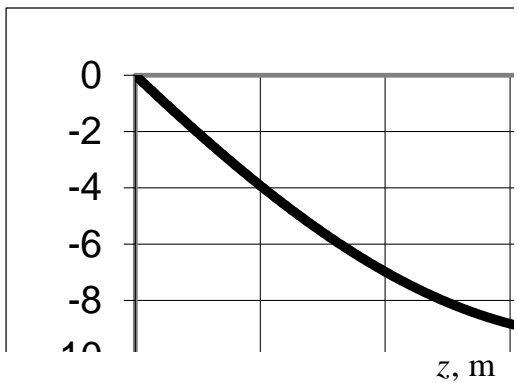

$u_{y}, \mathrm{~mm}$

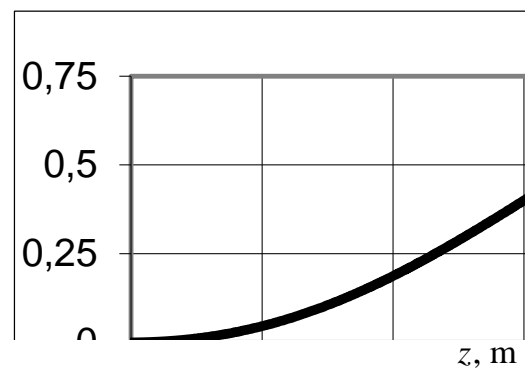

$u_{z}, \mathrm{~mm}$

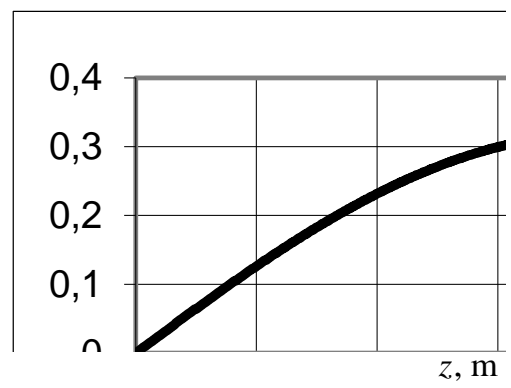

Fig. 5. The displacements and the rotations

For quantifying the role of the correction factors, the calculations are done without taking into account shear and cross links in the elasticity relations. 
Transverse forces, bending moments and deflections do not changed practically (less than $0,5 \%$ ), but axial displacements and rotations vanish.

Solving of the mode analysis problems with the calculation of the natural frequencies and the normal modes of free oscillations is not difficult. Here the frequency is considered as the additional variable (thirteenth): $\lambda^{2}=Y_{12}, D_{12}=0$.

\section{Conclusion}

So, the considering of the turbine blades as the rods is still advisable if there are no local peculiarities of the three-dimensional stress-strain state. New possibilities of computer mathematics allow us to calculate even the full inhomogeneous rod model with all of the compliances. Herewith in many cases ideas about the bending in two planes are enough.

\section{Acknowledgements}

This work is done as the part of the state task with the financial support of the Ministry of Education and Science of the Russian Federation (Project No. 9332014, 1972-2014).

\section{References}

1. Ilchenko BV, Gizzatullin RZ, Yarullin RR (2011) Stress-strain fields for blades of turbine K210-130 under operation loading. Treatises Akademenergo (Trudy Akademenergo) 3: 74-81 (in Russian)

2. Magerramova LA (2013) Increasing resource of the aviation gas turbine blades by calculation methods. Herald Mosc. Aviat. Inst. (Vestnik Moskovskogo Aviatsionnogo Instituta). 20(1): 58-70 (in Russian)

3. Melnikova GV, Shorr BF, Salnikov AV, Nigmatullin RZ (2014) Automated dynamic optimization of the blades of the gas turbine engines. Herald Mosc. Aviat. Inst. (Vestnik Moskovskogo Aviatsionnogo Instituta). 21(1): 76-85 (in Russian)

4. Trushin VA, Chechulin AYu (2012) Mathematical model of calculation of radial clearance between the working vanes and turbine housing. Herald Ufa State Aviat. Techn. Univ. (Vestnik UGATU). 16(2): 82-86 (in Russian)

5. Bloch HP, Singh MP (2009) Steam Turbines: Design, Applications and Relating. McGrawHill 433

6. Leyzerovich AS (2005) Wet-Steam Turbines for Nuclear Power Plants. PennWell 481

7. Brondsted P, Nijssen RPL (2013) Advances in wind turbine blade: design and materials. Woodhead Publishing Limited 484

8. Birger IA, Shorr BF et al. (1981) Dynamics of aviation gas turbine engines (Dinamika aviatsionnykh gazoturbinnykh dvigatelei). Mashinostroyenie, Moscow 232 (in Russian) 
9. Dimentberg FM, Kolesnikov, KS (1980) Vibrations in technics. Oscillations of machines, constructions and their elements (Vibratsiya v tehnike. Kolebaniya mashin, konstruktsiy $i$ ih elementov) Mashinostroyenie, Moscow 544 (in Russian)

10. Levin AV, Borishanskiy KN, Konson ED (1981) Strength and vibration of blades and disks of steam turbines (Prochnost i vibratsiya lopatok i diskov parovyih turbin). Mashinostroyenie, Leningrad 710 (in Russian)

11. Leonov VP, Schastlivaya IA, Igolkina TN et al. (2014) Application of finite element method for simulation of stress-strain state in manufacturing of long turbine blades made of highstrength titanium alloys. Inorganic Materials: Applied Research 5(6): 578-586 DOI $10.1134 / \mathrm{S} 2075113314060069$

12. Balakshin OB, Kukharenko BG, Khorikov AA (2007) Independent component analysis of oscillations under rotor blade flutter. Eng. and Autom. Problems 4: 77-83

13. Ganiev RF, Balakshin OB, Kukharenko BG (2009) On the occurrence of selfsynchronization of autooscillations of turbocompressor rotor blades. J Mach. Manuf. \& Reliability 38(6): 535-541 DOI 10.3103/S105261880906003X

14. Bezjazychny VF, Ganzen MA (2011) Estimation of blade-to-disk attachments deformation in turbomachines. Handbook. An Eng. J 4: 18-22 (in Russian)

15. Korihin NV (2009) Study of stress state of lock joint of ceramic blade of promising gas turbine. Russian J Heavy Mach. 1: 8-11 (in Russian)

16. Eliseev VV, Moskalets AA, Oborin EA (2015) Deformation analysis of turbine blades based on complete one-dimensional model. Russian J Heavy Mach. 5: 35-38 (in Russian)

17. Eliseev VV, Moskalets AA, Oborin EA (2015) Applying of Lagrange Equations to Calculation of Turbine Blade Vibration. Handbook. An Eng. J 8: 21-24 DOI 10.14489/hb.2015.08.pp.021-024 (in Russian)

18. Eliseev VV. (2003) Mechanics of elastic bodies. St. Petersburg State Polytechn. Univ. Publ. House, St Petersburg 336 (in Russian)

19. Kiryanov DV (2007) Mathcad 14. BHV-Peterburg, St Petersburg 704 (in Russian)

20. Dzhanelidze GYu (1946) Relations of Kirchhoff for naturally twisted rods and their applications (Sootnosheniya Kirchhoffa dlya estestvenno-zakruchennyih sterzhney $i$ ih prilozheniya). Treatises Leningrad Polytechn. Univ. (Trudy Leningradskogo Politechn. Univ.) 1: 23-32 (in Russian)

21. Vorobev YuS, Shorr BF (1983) Theory of twisted rods (Teoriya zakruchennyih sterzhney). Naukova Dumka, Kiev 188 (in Russian)

22. Eliseev VV (1991) Saint-Venant problem and elastic moduli for rods with curvature and twisting (Zadacha Saint-Venanta i uprugie moduli dlya sterzhney s kriviznoy i krucheniem). Mech. Solids. A Journal of Russian Acad. of Science (Izvestiya AN SSSR, MTT) 2: 167-176 (in Russian)

23. Iesan D (2008) Classical and generalized models of elastic rods. Chapman \& Hall 349 DOI $10.1201 / 9781420086508$

24. Khanh CL (1999) Vibrations of shells and rods. Springer 423 DOI 10.1007/978-3-64259911-8

25. Rubin MB (2000) Cosserat Theories: shells, rods and points. Springer 488 DOI 10.1007/97894-015-9379-3

26. Lurie AI (2005) Theory of elasticity. Springer, Berlin 1038 DOI 10.1007/978-3-540-26455-2 